\title{
NeuroImage
}

\section{Modeling the hemodynamic response to brain activation}

\author{
Richard B. Buxton, ${ }^{*}$ Kâmil Uludağ, David J. Dubowitz, and Thomas T. Liu \\ Department of Radiology, and Center for Functional MRI, University of California-San Diego, La Jolla, CA 92093-0677, United States
}

Available online 11 September 2004

\begin{abstract}
Neural activity in the brain is accompanied by changes in cerebral blood flow (CBF) and blood oxygenation that are detectable with functional magnetic resonance imaging (fMRI) techniques. In this paper, recent mathematical models of this hemodynamic response are reviewed and integrated. Models are described for: (1) the blood oxygenation level dependent (BOLD) signal as a function of changes in cerebral oxygen extraction fraction $(E)$ and cerebral blood volume (CBV); (2) the balloon model, proposed to describe the transient dynamics of $\mathrm{CBV}$ and deoxyhemoglobin (Hb) and how they affect the BOLD signal; (3) neurovascular coupling, relating the responses in $\mathrm{CBF}$ and cerebral metabolic rate of oxygen $\left(\mathrm{CMRO}_{2}\right)$ to the neural activity response; and (4) a simple model for the temporal nonlinearity of the neural response itself. These models are integrated into a mathematical framework describing the steps linking a stimulus to the measured BOLD and CBF responses. Experimental results examining transient features of the BOLD response (post-stimulus undershoot and initial dip), nonlinearities of the hemodynamic response, and the role of the physiologic baseline state in altering the BOLD signal are discussed in the context of the proposed models. Quantitative modeling of the hemodynamic response, when combined with experimental data measuring both the BOLD and CBF responses, makes possible a more specific and quantitative assessment of brain physiology than is possible with standard BOLD imaging alone. This approach has the potential to enhance numerous studies of brain function in development, health, and disease.

(c) 2004 Elsevier Inc. All rights reserved.
\end{abstract}

Keywords: Hemodynamic response; Brain activation; fMRI

\section{Introduction}

One of the remarkable developments in recent work on magnetic resonance (MR) imaging is the recognition that changes in the metabolic state of the brain affect the local MR signal and provide an intrinsic mechanism for detecting brain activation (Kwong et al., 1992; Ogawa et al., 1990). The origin of this effect

* Corresponding author. Department of Radiology, 0677 Center for Functional MRI, University of California-San Diego, 9500 Gilman Drive, La Jolla, CA 92093-0677. Fax: +1 8588220605.

E-mail address: rbuxton@ucsd.edu (R.B. Buxton).

Available online on ScienceDirect (www.sciencedirect.com.) is that hemoglobin $(\mathrm{Hb})$ is diamagnetic when oxygenated and paramagnetic when deoxygenated. The presence of deoxyhemoglobin alters the local magnetic susceptibility, creating magnetic field distortions within and around the blood vessels, and this microscopically inhomogeneous field produces a slight alteration in the local MR signal. If the local oxygen extraction fraction $(E)$ always remained constant, the deoxyhemoglobin concentration in blood would not change, and this biophysical effect would be only an interesting curiosity. However, when combined with an unexpected physiological phenomenon, this effect becomes a powerful tool for mapping brain activation. Following increased neural activity in the brain, the local cerebral blood flow (CBF) increases much more than the cerebral metabolic rate of oxygen $\left(\mathrm{CMRO}_{2}\right)$, and as a result $E$ decreases with activation (Fox and Raichle, 1986). Because the local blood is more oxygenated, there is less deoxyhemoglobin present, the magnetic field distortions are reduced, and the local MR signal increases slightly. This small blood oxygenation level dependent (BOLD) signal change is the mapping signal used in most functional magnetic resonance imaging (fMRI) applications.

Empirically, the BOLD effect has proven to be a sensitive tool for mapping brain activation. In a typical fMRI experiment at 1.5 $\mathrm{T}$, images are collected rapidly with a single-shot echo planar imaging (EPI) or spiral gradient echo acquisition with an echo time (TE) of about $40 \mathrm{~ms}$. The repetition time (TR) between subsequent images of a particular slice is about $2 \mathrm{~s}$, and dynamic images are acquired over several minutes as stimuli are presented to the subject. Then the time course of each image voxel is analyzed to detect BOLD signal time courses that show a significant correlation with the stimulus.

In such fMRI experiments, the goal is to map patterns of neuronal activation in the subject's brain while they perform specific tasks. However, the BOLD signal does not directly measure the neuronal activity itself. Instead, the BOLD effect is sensitive to the changes in $\mathrm{CBF}, \mathrm{CMRO}_{2}$, and cerebral blood volume (CBV), the set of physiological responses that are referred to collectively as the hemodynamic response to activation. A critical goal for interpreting fMRI data is to understand the underlying link between neuronal activity and the hemodynamic response.

In this paper, we review and integrate several recent models that have been proposed for describing different aspects of the 
hemodynamic response. Our goal is to develop a mathematical description of the translation from an applied stimulus pattern to the measured BOLD signal. Such a model necessarily includes speculative elements and should be regarded as a working model in need of experimental testing. A quantitative working model such as this can guide experimental design and inform the interpretation of experimental results. In particular, a modeling approach can help to identify possible sources of variability of the BOLD response across the brain and across subject populations, and provide mechanisms for how such variability can arise despite similar underlying neural responses.

The fact that the BOLD signal depends on a combination of changes in $\mathrm{CBF}, \mathrm{CBV}$, and $\mathrm{CMRO}_{2}$, and also on the baseline physiological state, makes it difficult to interpret the magnitude of the BOLD signal change unambiguously without further experimental information. For this reason, the BOLD effect has been used primarily as a mapping tool, based on detecting signal changes, rather than as a probe of the underlying physiology based on a detailed analysis of the BOLD response. However, when the BOLD signal change is combined with other MRI methods for measuring $\mathrm{CBF}$ directly, it becomes possible to untangle some of the factors that influence the BOLD effect, enabling a much more detailed modeling of the physiological processes.

Arterial spin labeling (ASL) techniques provide a means of measuring both the BOLD signal and CBF simultaneously (Buxton et al., 1998c; Wong et al., 1997). With ASL, the magnetization of arterial blood is inverted and, after a suitable delay to allow the tagged blood to enter the desired image plane, an image is acquired. The experiment is then repeated without tagging the arterial blood and another image is acquired as a control. The image difference, control minus tag, is then a direct reflection of how much arterial blood was delivered to each voxel and so provides a map of CBF. There are several variants of ASL techniques and several technical issues that must be addressed in order for the results to be interpreted in a quantitative fashion (see Buxton, 2002b for a review).

When these factors are taken into account, ASL offers a powerful probe of brain physiology. With a dual echo acquisition, with subsequent acquisitions alternating between tag and control, the data can be processed to yield essentially independent measurements of the local CBF and BOLD time series. Although such techniques are not yet widely available, they offer the best prospects for providing the data that will allow a quantitative interpretation of the BOLD effect. In addition, newer MRI techniques promise to provide measurements of $\mathrm{CBV}$ over time as well (Lu et al., 2003). In the following discussion of models of the hemodynamic response, we will assume that the measurable quantities are time series of $\mathrm{CBF}$ and BOLD, and that under some circumstances $\mathrm{CBV}$ can be measured as well.

Four models are considered, which when combined provide a model of the full path from a temporal stimulus pattern to a measured $\mathrm{CBF}$ response and a BOLD response. The models treat (1) the BOLD signal as a function of changes in $E$ and $C B V ;$ (2) the balloon model, proposed to describe the transient dynamics of CBV and deoxyhemoglobin and how they affect the BOLD signal; (3) neurovascular coupling, relating the responses in $\mathrm{CBF}$ and $\mathrm{CMRO}_{2}$ to the neural activity response; and (4) a simple model for the temporal nonlinearity of the neural response itself. Recent experimental findings on the linearity of the BOLD response and the effect of the baseline physiological state on the BOLD response are considered in light of these models.

\section{Experimental characterization of the hemodynamic response}

Based on numerous experimental studies of the BOLD and $\mathrm{CBF}$ responses to brain activation, the following are the key findings that motivate the modeling:

1. $\mathrm{CBF}$ increases much more than $\mathrm{CMRO}_{2}$ with brain activation, producing a reduction of $E$ and the total deoxyhemoglobin present in an image voxel (Fox and Raichle, 1986; Hoge et al., 1999). This phenomenon is the primary cause of the BOLD signal change.

2. The CBF and BOLD responses to even a very brief stimulus are delayed by $1-2 \mathrm{~s}$ and have a temporal width on the order of $4-6 \mathrm{~s}$ (Bandettini et al., 1992). For a sustained stimulus of $20 \mathrm{~s}$ or longer, the response typically reaches a plateau value, although there can be substantial variation (e.g., an initial overshoot, a slow ramp, or an overshot at the end of the stimulus).

3. A post-stimulus undershoot of the BOLD signal is common and may last for $30 \mathrm{~s}$ or more (Frahm et al., 1996; Kruger et al., 1996), with longer duration stimuli tending to have longer post-undershoots. The CBF response typically shows only a shorter and weaker post-stimulus undershoot, or none at all (Buxton et al., 1998c; Obata et al., 2004).

4. Some investigators have reported an initial dip of the BOLD signal lasting 1-2 s before the standard BOLD signal increase (Ernst and Hennig, 1994; Hu et al., 1997; Menon et al., 1995; Yacoub and $\mathrm{Hu}, 2001$ ), and a corresponding transient increase of deoxyhemoglobin has been reported in optical imaging studies (Malonek and Grinvald, 1996). The effect is small and not always present (Buxton, 2001; Jones et al., 2001; Lindauer et al., 2001), but it has stirred interest because it may reflect a rapid increase of $\mathrm{CMRO}_{2}$ before the $\mathrm{CBF}$ increase, and this phenomenon may be better localized to the area of increased metabolism (i.e., the CBF increase may cover a wider area) (Malonek and Grinvald, 1996).

5. The BOLD response typically exhibits a temporal nonlinearity such that an appropriately shifted and added response to a brief stimulus over-predicts the true response to an extended stimulus (Birn et al., 2001; Boynton et al., 1996; Friston et al., 1998; Glover, 1999; Miller et al., 2001; Robson et al., 1998; Vasquez and Noll, 1998). This temporal nonlinearity is most pronounced when the brief stimulus is less than about $4 \mathrm{~s}$ and the extended stimulus is longer than $6 \mathrm{~s}$. Comparing short and long duration stimuli that are both longer than about $4 \mathrm{~s}$, the temporal nonlinearity is reduced.

6. Nonlinearity has also been reported as a "refractory period", such that two identical stimuli presented close together in time produce a net response with less than twice the integrated response of a single stimulus alone (Boynton et al., 1996; Buckner, 1998; Huettel and McCarthy, 2001).

7. There is a growing body of evidence suggesting that the baseline CBF can have a strong effect on the magnitude of the BOLD response to the same stimulus (Corfield et al., 2001; Davis et al., 1998; Hoge et al., 1999; Kastrup et al., 1999, 2002; Kim et al., 1999; Li et al., 1999). For example, if baseline $\mathrm{CBF}$ is increased by breathing $\mathrm{CO}_{2}$, the BOLD response to the same task is reduced substantially. Interestingly, however, the $\mathrm{CBF}$ change $(\triangle \mathrm{CBF})$ appears to remain the same despite the baseline change (Ances et al., 2001; Kastrup et al., 2002; Li et al., 1999, 2000). Similar results have been found with injection of acetazolamide, a carbonic anhydrase 
inhibitor (Brown et al., 2003). Carbonic anhydrase catalyzes the conversion of $\mathrm{CO}_{2}$ to bicarbonate ions, which increases the carrying capacity of the blood for removing $\mathrm{CO}_{2}$. Inhibiting this enzyme may lead to increased $\mathrm{CO}_{2}$ concentration in the brain, so the mechanism of action for increasing CBF may be the same as for $\mathrm{CO}_{2}$ itself.

With these examples as experimental background, we now consider quantitative models for the hemodynamic response.

\section{Definition of dynamic variables}

For the remainder of the paper we will use the more compact notation defined in Table 1 for the dynamic variables and parameters. The assumed causal connections between the variables are diagrammed in Fig. 1: (1) the stimulus pattern $s(t)$ drives the neural response $N(t)$; (2) $N(t)$ drives the CBF response $f(t)$ and the $\mathrm{CMRO}_{2}$ response $m(t)$; (3) $f(t)$ and $m(t)$ drive the balloon model to produce the CBV response $v(t)$ and the total deoxyhemoglobin response $q(t)$; and (4) $q(t)$ and $v(t)$ combine to produce the BOLD signal. In most cases we use the convention that upper case variables refer to absolute quantities, while lower case variables are the same quantity normalized to its baseline value. Then, for example, at baseline $f=m=q=v=1$ and $E=E_{0}$.

For the calculations shown here, we are particularly interested in transient features and nonlinearities of the BOLD response. To emphasize these effects, we assume simple forms for scaling the stimulus and the neural response. The stimulus is considered to be a brief event (e.g., one reversal of a visually presented checkerboard), and these events can be presented in any pattern, including direct concatenation to produce a sustained stimulus (e.g., a flickering checkerboard). The stimulus pattern $s(t)$ is then a time series of ones and zeroes defining when events occurred. The neural response is defined such that $N(t)=1$ on the plateau of a sustained stimulus when no adaptation effects are operating.

\section{Physiological relationships}

The CBF increase associated with neural activity is triggered by a relaxation of the smooth muscle in the wall of the arterioles. The arterioles provide most of the resistance in the vascular tree and provide a way to quickly decrease vascular resistance by relaxing. As the resistance of the arterioles decreases, the pressure drop across these vessels also decreases, raising the pressure in the capillaries and veins. These vessels may also expand due to the increased pressure, further increasing the CBV. Experimental studies (Grubb et al., 1974) have indicated that the steady-state relationship between $\mathrm{CBF}$ and $\mathrm{CBV}$ can be described with a power law:

$v=f^{\alpha}$

where the exponent is approximately $\alpha=0.4$. This empirical relationship applies to the entire blood volume. For our purposes we are really interested in the venous volume, and to a lesser extent the capillary volume, because this is where the deoxyhemoglobin lies. Nevertheless, a value of $\alpha=0.4$ is often used in modeling the BOLD effect.
Table 1

Model variables, parameters, and typical values

\begin{tabular}{ll}
\hline & Definitions \\
\hline $\begin{array}{l}\text { Dynamic variables } \\
f(t)\end{array}$ & $\mathrm{CBF}$ normalized to baseline \\
$m(t)$ & $\mathrm{CMRO}_{2}$ normalized to baseline \\
$v(t)$ & $\mathrm{CBV}$ normalized to baseline \\
$q(t)$ & DeoxyHb content normalized \\
& to baseline \\
$b(t)$ & BOLD signal change $(\%)$ \\
$E(t)$ & $\mathrm{O}_{2}$ extraction fraction \\
$N(t)$ & Neural activity \\
$s(t)$ & Stimulus pattern
\end{tabular}

Physiological parameters $F_{0}\left(0.01 \mathrm{~s}^{-1}\right)$

$\Delta F$

$E_{0}(0.4)$

$V_{0}(0.03)$

$\alpha(0.4)$

$n(2-3)$

Baseline $\mathrm{CBF}\left(0.01 \mathrm{~s}^{-1}=\right.$ $60 \mathrm{ml} \mathrm{min}^{-1} \mathrm{ml}^{-1}$ tissue) Absolute CBF change with activation

Baseline $\mathrm{O}_{2}$ extraction fraction Baseline blood volume Steady state flow-volume relation: $v=f^{\alpha}$

Steady-state flow-metabolism relation: $n=(f-1) /(m-1)$

$B O L D$ signal parameters

$a_{1}(3.4)$

Weight for deoxyHb change Weight for blood volume change (estimated for $1.5 \mathrm{~T}$ )

Balloon model parameters

$f_{\text {out }}(v, t)$

Outflow from the balloon (transiently different from $f$ )

$\tau_{\mathrm{MTT}}$

$\tau_{+}(0-30 \mathrm{~s})$

$\tau_{-}(0-30 \mathrm{~s})$

Transit time through the balloon $\left(V_{0} / F_{0}\right)$

Viscoelastic time constant (inflation)

Viscoelastic time constant (deflation)

Neural response parameters

$\kappa_{n}(0.0-2.0)$

Inhibitory gain factor

$\tau_{i}(1-3 \mathrm{~s})$

Inhibitory time constant

Neurovascular coupling (assumed linear) $\tau_{\mathrm{f}}(4 \mathrm{~s})$

$\tau_{\mathrm{m}}(4 \mathrm{~s})$

$\delta t(0-2 \mathrm{~s})$

$f_{1}(1.0-2.0)$

Width of CBF impulse response

Width of $\mathrm{CMRO}_{2}$ impulse response

Delay of CBF relative to $\mathrm{CMRO}_{2}$ responses

Normalized CBF response to sustained neural activation

At steady-state, $\mathrm{CBF}$ and $\mathrm{CMRO}_{2}$ are related to each other by the arterial oxygen concentration $C_{\mathrm{a}}$ and the net oxygen extraction fraction $E$ :

$\mathrm{CMRO}_{2}=E \cdot C_{\mathrm{a}} \cdot \mathrm{CBF}$

$m=\frac{E}{E_{0}} f$

The local oxygenation of the venous blood depends directly on $E$. 


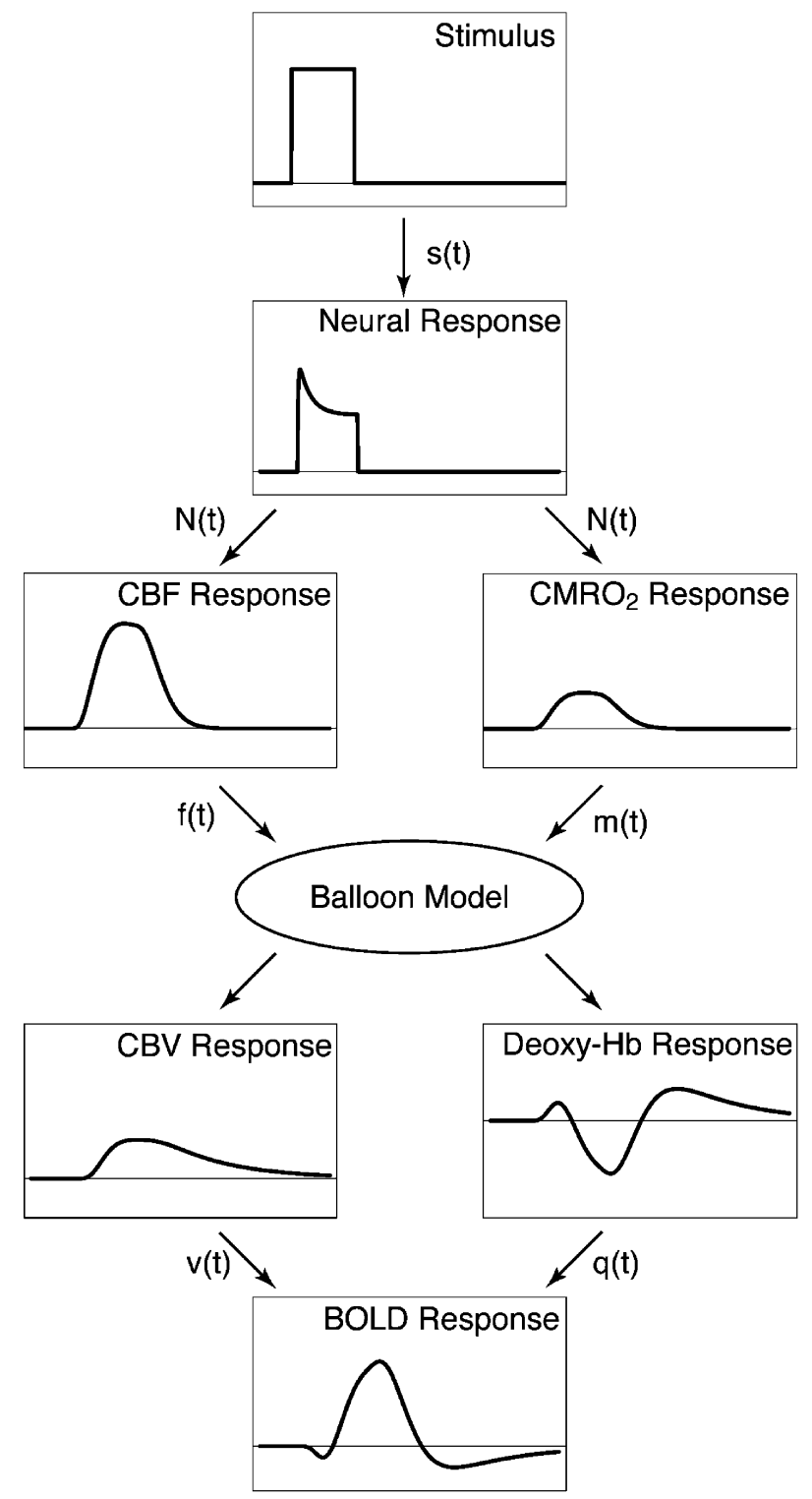

Fig. 1. Diagram of the proposed model linking the applied stimulus to the resulting physiological responses and the measured BOLD response.

For modest changes around an awake baseline state, experiments suggest that the relationship between the $\mathrm{CBF}$ and $\mathrm{CMRO}_{2}$ changes can be characterized as linear with a slope $n$ defined as the fractional change in CBF divided by the fractional change in $\mathrm{CMRO}_{2}$ :

$n=\frac{\Delta \mathrm{CBF} / \mathrm{CBF}_{0}}{\Delta \mathrm{CMRO}_{2} / \mathrm{CMRO}_{20}}$

$n=\frac{f-1}{m-1}$

where the subscript " 0 " denotes baseline values. Most recent experimental measurements find $n=2-3$ (Davis et al., 1998; Hoge et al., 1999; Kastrup et al., 2002; Marrett and Gjedde, 1997; Seitz and Roland, 1992), although larger values have also been reported (Fox and Raichle, 1986; Kuwabara et al., 1992).

The fact that $n>1$, so that $E$ decreases with activation, is the physiological source of the BOLD effect, and this was originally described as an uncoupling of $\mathrm{CBF}$ and $\mathrm{CMRO}_{2}$ in the seminal work of Fox and Raichle (1986). However, a promising alternative explanation developed over the last few years is the oxygen limitation model (Buxton, 2002a; Buxton and Frank, 1997; Gjedde et al., 1991, 1999; Hyder et al., 1998). By this model, the drop in $E$ with activation plays a functional role, rather than serving as a marker of an uncoupling. The key idea of this model is to think of the $\mathrm{O}_{2}$ flux down a diffusion gradient from the mean capillary value to the mean value in mitochondria. To increase the net flux (i.e., increase $\mathrm{CMRO}_{2}$ ), the gradient must be increased. If there is no capillary recruitment, so the $\mathrm{O}_{2}$ source cannot be brought closer to the mitochondria, and the mean mitochondrial $p \mathrm{O}_{2}$ is very low, then the only way to increase the $\mathrm{O}_{2}$ flux is to raise the average capillary $p \mathrm{O}_{2}$. The average capillary $p \mathrm{O}_{2}$ lies somewhere between the arterial and venous $p \mathrm{O}_{2}$ values, and because the arterial $p \mathrm{O}_{2}$ is fixed, the only local control available is to raise the venous $p \mathrm{O}_{2}$. And raising venous $p \mathrm{O}_{2}$ requires a reduction of the oxygen extraction fraction $E$. In this way, the decrease in $E$ is necessary to increase the $\mathrm{O}_{2}$ diffusion gradient from capillaries to mitochondria. For the calculations in this paper, we will simply use the empirical relationship in Eq. (3).

Eqs. ((1)-(3)) are useful for relating the key physiological quantities involved in the BOLD effect, but it is important to remember that while Eq. (2) is necessarily true from the definition of the terms involved, Eqs. (1) and (3) are empirical relationships, and the uniformity of these relationships across brain regions, subject populations, species, and different physiological states is still open to question and in need of experimental evaluation.

\section{Modeling the BOLD effect}

The BOLD effect is primarily due to changes in local deoxyhemoglobin content, but quantitative modeling of this effect requires some subtlety. In fact, there are two sources of signal change that must be modeled: the intravascular and the extravascular signals (Boxerman et al., 1995a; Ogawa et al., 1993). Both regimes are affected by the magnetic field gradients created by the presence of deoxyhemoglobin, which cause the MR signal to decay faster when deoxyhemoglobin increases. Although the intrinsic intravascular signal is much less than the extravascular signal, the sensitivity of the intravascular signal to the oxygenation of blood is much greater. The result is that the intravascular contribution likely accounts for half or more of the signal change observed at $1.5 \mathrm{~T}$. The total deoxyhemoglobin content could change either by changing the oxygen extraction fraction or by changing the volume of the venous blood, so the role of volume changes must be included (Boxerman et al., 1995b; Ogawa et al., 1993; Yablonsky and Haacke, 1994). And finally, for the smallest vessels, diffusion effects can be important. Thus modeling the BOLD effect depends not only on the biophysical models for how intravascular susceptibility differences alter the signal, but also physiological models for how $\mathrm{CBF}, \mathrm{CBV}$, and $\mathrm{CMRO}_{2}$ change with activation. The relative changes in $\mathrm{CBF}$ and $\mathrm{CMRO}_{2}$ determine the level of oxygenation of the blood, and the CBV determines the total amount of blood (and thus the total deoxyhemoglobin present in the voxel).

\section{Magnetic susceptibility effects and the MR signal}

Ogawa et al. (1993) introduced a biophysical model for the BOLD effect and Davis et al. (1998) extended this model based on 
reasonable approximations and the results of numerical simulations. Because of its simplicity, the model has proven to be a useful tool for understanding the BOLD effect in a quantitative way and has provided a method for calibrating the BOLD signal and measuring $\mathrm{CMRO}_{2}$ changes. The model starts from the simple picture of how the BOLD effect arises, and relates the signal change to the underlying physiological variables and a few parameters that describe the local tissue.

The MR signal for a typical gradient echo acquisition is modeled as a simple exponential dependence on the echo time TE and can be written as:

$$
\begin{aligned}
& S=S_{\max } \cdot e^{-\mathrm{TE} \cdot R_{2}^{*}} \\
& R_{2}^{*}=R_{2}^{*}(0)+R
\end{aligned}
$$

where $S_{\max }$ is the effective spin density (the signal that would be measured if TE could be reduced to zero). The transverse relaxation rate constant $R_{2} *$ is written as a sum of two terms: $R_{2} *(0)$ is the value of $R_{2} *$ if no deoxyhemoglobin is present, and $R$ describes the additional relaxation produced by deoxyhemoglobin. Note that typically $R_{2} *(0)$ is much larger than $R$, that is, the local $T_{2} *$ that describes the decay of the signal is largely determined by the intrinsic $T_{2}$ and large-scale field gradients through the voxel, and the additional effect of deoxyhemoglobin is minor. For this reason, the signal changes due to the BOLD effect are small, but measurable.

We now assume that with activation $R$ is the only parameter that changes. Using the subscript " 0 " to denote the resting value and "act" to denote the activated value, the BOLD signal change with activation $\Delta S=S_{\text {act }}-S_{0}$ is:

$$
\begin{aligned}
\frac{\Delta S}{S_{0}} & =e^{-\Delta R_{2}^{*} \cdot \mathrm{TE}}-1 \approx-\Delta R_{2}^{*} \cdot \mathrm{TE} \\
\Delta R_{2}^{*} & =R_{\mathrm{act}}-R_{0}
\end{aligned}
$$

The key question is: how does $\Delta R_{2}{ }^{*}$ depend on blood oxygenation and volume? The magnitude of the magnetic field distortions near a magnetized vessel is proportional to the magnetic susceptibility difference between the blood and the surrounding extravascular space. Experiments indicate that the magnetic susceptibility difference can be accurately modeled as having a linear dependence on the local deoxyhemoglobin concentration in blood, and this quantity in turn can be expressed in terms of the change in the oxygen extraction fraction $E$.

However, the scaling of the field offsets does not necessarily define the scaling of the signal attenuation. As spins evolve in an inhomogeneous field, a distribution of phase angles develops, and it is this phase dispersion at the time of data collection that determines $R$. In particular, diffusion of water molecules effectively smooths the field distribution to create a narrower spread of phases. To model this in an approximate way, Davis et al. assumed a power law relationship between $R$ and $\Delta B$, the magnitude of the field distortions: $R \propto \Delta B^{\beta}$. Numerical simulations (Boxerman et al., 1995a,b; Ogawa et al., 1993) and theoretical analyses (Yablonsky and Haacke, 1994) suggest that when diffusion is not important $\beta \approx 1$, but that $\beta \approx 2$ gives a better description around the smallest vessels where diffusion effects are important. Numerical simulations for a mixture of vessel sizes suggest that $\beta=1.5$ is a good approximation for $1.5-3 \mathrm{~T}$, but that at higher fields $\beta$ should approach 1 .

In addition to the change in $E$ with activation, a change in blood volume also affects $R$. For example, even if the oxygenation of the blood did not change but the venous blood volume increased, the total deoxyhemoglobin would be increased, and we would expect this to increase $R$ and decrease the net MR signal. Numerical simulations suggest that a reasonable approximation is to assume that $R$ is proportional to $V$, the venous blood volume. Combining these dependences, the contribution of deoxyhemoglobin to the relaxation rate is modeled as:

$R \propto V E^{\beta}$

\section{The BOLD signal change}

Following Davis et al. (1998), these ideas can be combined to model the MR signal in terms of the blood volume $(V)$ and the oxygen extraction fraction $(E)$ :

$\frac{\Delta S}{S_{0}} \approx A \cdot\left[1-\frac{V_{\mathrm{act}}}{V_{0}}\left(\frac{E_{\mathrm{act}}}{E_{0}}\right)^{\beta}\right]$

The parameter $A$ lumps together TE and the unknown proportionality constant in Eq. (6), and is also proportional to the local resting blood volume $V_{0}$ and the resting oxygen extraction fraction $E_{0}^{\beta}$. A decrease of either of the physiological quantities ( $V$ or $E$ ) will decrease the local deoxyhemoglobin concentration and so increase the MR signal.

Eq. (7) for the BOLD signal change is quite simple, depending on two physiological changes (the change in blood volume $V$ and oxygen extraction fraction $E$ ) and two additional parameters $\beta$ and $A$. The form of the signal equation directly describes the ceiling effect on the BOLD signal. In simple terms, $A$ is the maximum BOLD signal change that could occur, corresponding to complete removal of deoxyhemoglobin from the voxel. The parameter $\beta$ should be primarily field dependent, and we can assume that it is not a function of brain region. The parameter $A$, however, is a local parameter and so may vary across different voxels in the brain. Note that this parameter is proportional to the value of $R$ at rest, the relaxation rate produced by deoxyhemoglobin in the baseline state. This means that the more deoxyhemoglobin is present at rest, the larger the BOLD signal change will be for the same fractional change in $V$ and $E$ with activation. We will come back to this later when we consider the effect of the baseline condition on the magnitude of the BOLD effect.

In our notation with dynamic variables normalized to their baseline values, and assuming Eq. (1) is accurate, the basic BOLD signal equation is:

$\frac{\Delta S}{S_{0}}=A \cdot\left(1-f^{\alpha-\beta} m^{\beta}\right)$

Although Eq. (8) is a very useful model, the reader should bear in mind that it does not necessarily describe all of the effects that may contribute to the measured signal change in an activation experiment. Specifically, small direct effects of $\mathrm{CBF}$ and $\mathrm{CBV}$ changes on the MR signal that are independent of the BOLD effect are likely present in real data. For example, if the repetition time TR is shorter than the $T_{1}$ of blood and the flip angle is large (e.g., $90^{\circ}$ ), the increased delivery of fresh unsaturated blood due to increased CBF could increase the net signal slightly. In addition, the intrinsic signal from arterial blood typically is larger than the intrinsic signal of the extravascular space at $1.5 \mathrm{~T}$, so increasing the arterial blood volume fraction of the voxel could also produce 
a slight signal increase. Note that both of these effects are due to arterial blood changes, where deoxyhemoglobin is negligible, so these are effects in addition to the BOLD effect. In most applications, these effects are thought to be small compared to the BOLD effect, especially at higher magnetic fields, but they may not be negligible.

\section{Calibrated BOLD approach for measuring $\mathrm{CMRO}_{2}$ changes}

Davis et al. (1998) showed that by measuring both the BOLD signal change and the CBF change with activation, and analyzing these data in terms of the BOLD signal model, it is possible to estimate the change in $\mathrm{CMRO}_{2}$. This technique was used effectively by Hoge et al. (1999) to explore the coupling of CBF and $\mathrm{CMRO}_{2}$ during brain activation. The essential problem in applying Eq. (8) to measured data is the uncertainty about the local value of $A$. If $A$ is known, then $m$ can be determined from Eq. (8) when $f$ and $\Delta S$ are measured with an ASL experiment. To measure $A$, Davis et al. and others have exploited a well-established-but poorly understood-physiological phenomenon: breathing $\mathrm{CO}_{2}$ significantly raises $\mathrm{CBF}$ but has little or no effect on $\mathrm{CMRO}_{2}$. This provides a way to calibrate the BOLD signal with a hypercapnia experiment. By measuring $f$ and $\Delta S$ in response to breathing $\mathrm{CO}_{2}$, combined with the assumption that $\mathrm{CMRO}_{2}$ remains constant $(m=$ 1 ), the value of $A$ is calculated from Eq. (8). The same equation is applied again to the measured activation signals in that region, and with a known value of $A$, the value of $m$ with activation can be calculated. Because of the assumptions involved (Eq. (1)), this is essentially a steady-state measurement of $\mathrm{CMRO}_{2}$ change from baseline. However, if both $\mathrm{CBF}$ and $\mathrm{CBV}$ time courses are measured independently, a dynamic curve for $\mathrm{CMRO}_{2}$ can be calculated (Mandeville et al., 1999a).

\section{Alternative forms for the BOLD signal model}

An alternate form of the BOLD signal equation was proposed to model the dynamics of the BOLD effect in the context of the balloon model (Buxton et al., 1998c) (described in the next section). The derivation of this model is based on separate estimates of the intravascular and extravascular signal changes. In this way, the model can be used to analyze experiments in which flow-nulling bipolar gradient pulses are applied to destroy the signal of moving blood, and thus eliminate the intravascular signal changes from the BOLD effect (Buxton et al., 1998a). The key physiological variables are the total deoxyhemoglobin $(q)$ and the blood volume $(v)$, both normalized to their values at rest. In this model, the BOLD signal change is written as:

$\frac{\Delta S}{S} \approx V_{0}\left[a_{1}(1-q)-a_{2}(1-v)\right]$

where $V_{0}$ is the resting venous blood volume fraction (e.g., 0.03) and the dimensionless parameters $a_{1}$ and $a_{2}$ depend on several experimental and physiological parameters. The values estimated by Obata et al. (2004) for a magnetic field of $1.5 \mathrm{~T}$ with $\mathrm{TE}=40$ $\mathrm{ms}$ and $E_{0}=0.4$ are $a_{1}=3.4$ and $a_{2}=1.0$.

Eqs. (8) and (9) are framed in terms of different variables, but they are approximately equivalent expressions for the BOLD signal change. Fig. 2 shows curves of the BOLD response as a function of

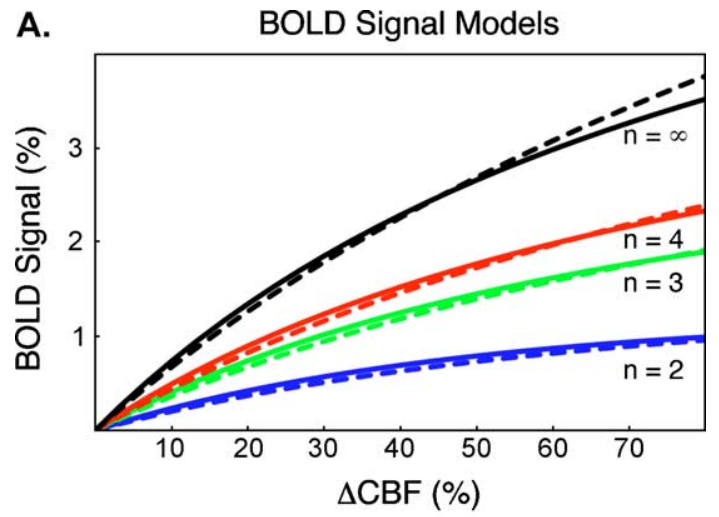

B. Signal Trajectory

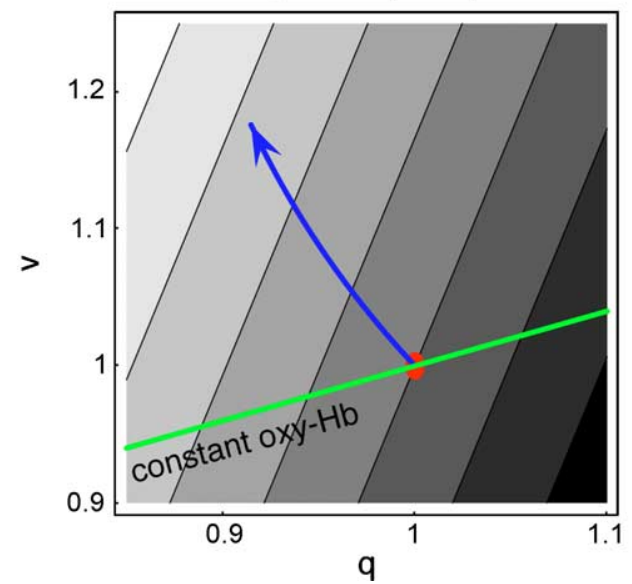

Fig. 2. (A) The modeled BOLD signal as a function of the CBF change with activation illustrating the approximate equivalence of the model in Eq. (8) (with $A=0.075$ and $\beta=1.5$, solid lines) and Eq. (9) (with $V_{0}=0.03$, dashed lines). Curves are shown for several values of the $\mathrm{CBF} / \mathrm{CMRO}_{2}$ coupling parameter $n$. The curvature of these lines is due to the BOLD ceiling effect, and this is an important source of nonlinearity in the BOLD response. (B) Steady-state trajectory for a CBF increase up to $50 \%$ with $n=$ 3 plotted in the $q / v$ plane, where $q$ is normalized total deoxyhemoglobin and $v$ is normalized venous blood volume. Contours are the BOLD signal in steps of $0.5 \%$, calculated from Eq. (9). The initial baseline value $(q=v=1)$ is shown as a red dot, and a line of constant oxy-Hb is shown in green.

the CBF change calculated with the two models with $A=0.075$ in Eq. (8) and $V_{0}=0.03$ in Eq. (9) (other parameters were the standard values listed in Table 1$)$. These curves were calculated for steady-state changes using the relation $q / v=E / E_{0}$ to relate the variables of the two equations. In their calibrated BOLD experiments in human visual cortex, Davis et al. found an average value of $A=0.079$, and $3 \%$ is a reasonable estimate of the venous blood volume fraction. This suggests that the theoretical assumptions that led to the estimates of $a_{1}$ and $a_{2}$ in Eq. (9) are in reasonable agreement with experimental data, and the similarity of the curves illustrates the consistency of the two models.

Eq. (8) is useful for calibrated BOLD studies, because it explicitly includes CBF, a measurable quantity. On the other hand, Eq. (9) deals explicitly with the variables of the balloon model, and so is more convenient for most of the modeling calculations described in this paper.

Based on Eq. (9), we can look at the BOLD signal as a contour plot in the $q / v$ plane, because the dynamic variables $q$ 
and $v$ determine the signal. Fig. 2B shows such a plot, along with a curve of constant oxyhemoglobin (a line of constant deoxyhemoglobin is vertical). Note that the BOLD signal does not purely follow total deoxyhemoglobin or oxyhemoglobin (i.e., the contours of constant BOLD signal are neither horizontal nor parallel to the constant oxyhemoglobin curve). Because of this, it is possible for the BOLD signal to increase despite an increase of deoxyhemoglobin. As an example, consider a scenario in which the increase in $\mathrm{CBV}$ and the decrease in $E$ happen to combine to produce no change in total deoxyhemoglobin, so that $q=1$ but $v>1$. In this case, the field distortions outside the vessels will be similar, but the concentration of deoxyhemoglobin in the blood must have decreased. The intravascular signal depends strongly on the deoxyhemoglobin concentration, and so this component of the signal will increase. The result is a BOLD signal increase with no change in deoxyhemoglobin. This subtlety of the BOLD signal may become important in comparing fMRI data with optical or near-infrared results that are sensitive to deoxyhemoglobin and oxyhemoglobin: there is no one-to-one correspondence between the BOLD signal and the total deoxyhemoglobin (Hess et al., 2000).

Analyzing the BOLD signal in the $q / v$ plane is also useful for visualizing the physiological dynamics accompanying brain activation. Fig. 2B shows a simple trajectory that would result for a gradual $\mathrm{CBF}$ increase of $50 \%$ with $n=3$, if the physiological quantities all followed their steady-state relationships (Eqs. ((1)(3))) at all times. The interesting dynamics develops when the physiological variables transiently depart from these relationships in the transition to a new steady-state, and then the trajectories become rather more complicated. The following models attempt to describe these dynamics.

\section{The balloon model}

The balloon model (Buxton et al., 1998c) was motivated by the observation in an animal study (Mandeville et al., 1998) that CBV returned to baseline more slowly than $\mathrm{CBF}$ after the end of the stimulus, and the idea that this effect might explain the poststimulus undershoot of the BOLD signal that is often observed. A similar windkessel model was proposed by Mandeville et al. (1999b) to embody the same concept and provide a biomechanical mechanism for a delayed CBV return to baseline. The balloon model has been refined and compared with experimental data (Feng et al., 2001; Friston, 2002; Friston et al., 2000; Mildner et al., 2001; Obata et al., 2004; Toronov et al., 2003), and some errors in the original parameter estimates were recently corrected (Obata et al., 2004). The model is capable of producing BOLD poststimulus undershoots that match well with experimental data. However, the central premise of the model, that the undershoot occurs when CBV returns slowly to baseline, has not been definitively established and focused experimental tests of this question are needed (e.g., Mandeville et al., 1999a; Toronov et al., 2003).

The central idea of the model is that the venous compartment is treated as a distensible balloon. The inflow to the balloon $f_{\text {in }}$ is the cerebral blood flow ( $f$ in our current notation), while the outflow from the balloon $f_{\text {out }}$ is an increasing function of the balloon volume. The two dynamical variables are the total deoxyhemoglobin $q(t)$ and the volume of the balloon $v(t)$. The equations of the balloon model represent mass conservation for blood and deoxyhemoglobin as they pass through the venous balloon:

$\frac{\mathrm{d} q}{\mathrm{~d} t}=\frac{1}{\tau_{\mathrm{MTT}}}\left[f(t) \frac{E(t)}{E_{0}}-\frac{q(t)}{v(t)} f_{\text {out }}(v, t)\right]$

$\frac{\mathrm{d} v}{\mathrm{~d} t}=\frac{1}{\tau_{\mathrm{MTT}}}\left[f(t)-f_{\text {out }}(v, t)\right]$

The net extraction fraction of oxygen is $E(t)$, and the resting value is typically $E_{0}=0.4$. The time dimension of the equations is scaled by the time constant $\tau_{\mathrm{MTT}}$, the mean transit time through the balloon at rest. For a cerebral blood flow of $60 \mathrm{ml} \mathrm{min}^{-1} 100 \mathrm{ml}^{-1}$ of tissue (equivalent to a rate constant of $0.01 \mathrm{~s}^{-1}$ ) and a resting venous blood volume fraction of $V_{0}=0.03$, the mean transit time is $\tau_{\mathrm{MTT}}=3 \mathrm{~s}$.

The driving function of the system is the quantity $f(t) E(t)$. In the original formulation of the balloon model, the extraction fraction was modeled as a fixed function of the inflow $f$, a tight coupling of flow and oxygen metabolism. The equations were generalized by Obata et al. (2004), treating $E(t)$ as an independent quantity to be able to explore the dynamics that result from uncoupling of blood flow and oxygen metabolism. Note that the quantity $f E / E_{0}$ is simply the cerebral metabolic rate of oxygen $\left(\mathrm{CMRO}_{2}\right)$ normalized to its value at rest $(\mathrm{m})$.

In the original formulation of the balloon model (Buxton et al., 1998c), the outflow was modeled as a pure function of blood volume v. Steady-state experiments (Grubb et al., 1974), altering CBF with inhaled $\mathrm{CO}_{2}$, found that the steady-state relationship between $\mathrm{CBF}$ and total blood volume was well described by an empirical power law (Eq. (1)). However, interesting dynamics occur when the blood volume transiently lags behind this steady-state relationship, for example, due to viscoelastic effects. In the original discussion of the balloon model, the description of these transients was an arbitrary mathematical form, chosen just to illustrate the type of effects that could occur. However, that approach is not well suited to data modeling. In particular, it would be useful to have a simple model that could be tested against multiple data sets, such as experiments varying the duration of the stimulus.

To that end, we proposed a simple model for these viscoelastic effects in which $f_{\text {out }}$ is treated as a function of the balloon volume and the rate of change of that volume (Buxton et al., 1998b):

$f_{\text {out }}(v)=v^{\frac{1}{\alpha}}+\tau \frac{\mathrm{d} v}{\mathrm{~d} t}$

With this form, the balloon initially resists a change in volume, but eventually settles into a new steady-state that conforms with the power law model in Eq. (1). The time constant $\tau$ controls how long this transient adjustment requires. A nonzero value for $\tau$ produces hysteresis in the curve $f_{\text {out }}(v)$, so that the system follows a different curve on inflation and deflation. To generalize this form and enable more fine tuning to data, we allow $\tau$ to take on different values during inflation $\left(\tau_{+}\right)$and deflation $\left(\tau_{-}\right)$.

For a specified driving function $f(t) E(t)$, and values for the parameters $\tau_{\mathrm{MTT}}, E_{0}, \alpha, \tau_{+}$, and $\tau_{-}$, Eqs. (10) and (11) can be integrated numerically to yield dynamic time courses for $q(t)$ and $v(t)$. These dynamic physiological quantities can then be combined with the BOLD signal model (Eq. (9))) to generate MR signal curves. Fig. 3 shows balloon model curves for a simple smooth trapezoidal form for $f(t)$ and a fixed $\mathrm{CBF} / \mathrm{CMRO}_{2}$ coupling parameter $n=3$. A nonzero value for $\tau_{+}$creates an initial overshoot of the BOLD signal, and a nonzero value for $\tau_{-}$creates 
Balloon Model Calculations
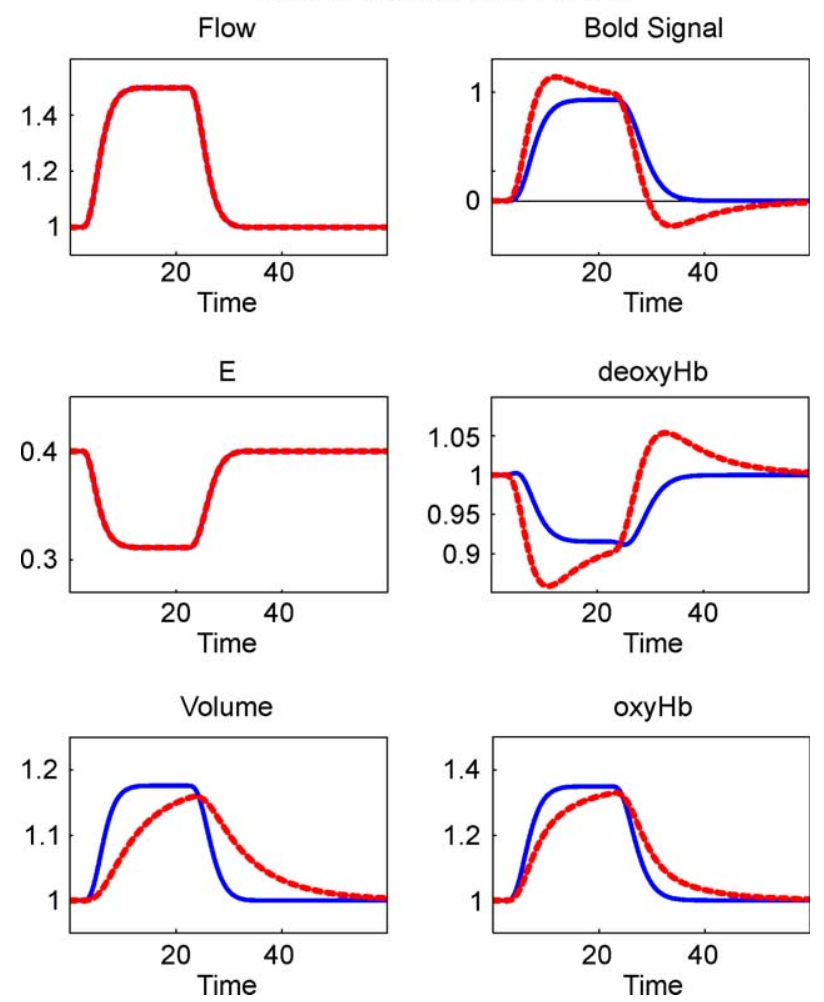

Fig. 3. Dynamic curves calculated with the balloon model for two sets of model parameters: $\tau_{+}=\tau_{-}=0$ (blue solid curve) and $\tau_{+}=\tau_{-}=20$ (red dashed curve). The input curves for inflow $f(t)$ ("Flow") and oxygen extraction fraction ("E") were identical for both calculations. Nonzero values for $\tau_{+}$or

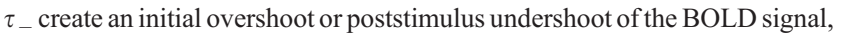
respectively, by causing the $\mathrm{CBV}$ to change more slowly than $\mathrm{CBF}$.

a poststimulus undershoot. These curves show that quite different BOLD responses can result from the same underlying $\mathrm{CBF}$ and $\mathrm{CMRO}_{2}$ response.

\section{Neurovascular coupling}

We do not currently have a quantitative understanding of the mechanisms that couple neural activity to $\mathrm{CBF}$ and $\mathrm{CMRO}_{2}$ changes. In fact, there is no consensus on exactly which aspect of neural activity drives the hemodynamic response, and this is an active area of research. Experimental studies comparing electrophysiological measurements with BOLD and CBF changes have found that the hemodynamic responses correlate better with local mean field potential, rather then local spiking rates, suggesting that the hemodynamic response is dominantly driven by input synaptic activity rather than output spiking activity (Lauritzen, 2001; Lauritzen and Gold, 2003; Logothetis, 2002; Logothetis et al., 2001). Theoretical analyses of the energy budget for neuronal signaling provide some support for this picture as well (Attwell and Laughlin, 2001; Lennie, 2003). The primary expenditure of energy is required to restore the ion gradients degraded during neural activation. The intracellular-extracellular sodium gradient is far from equilibrium, so pumping sodium against this gradient is a strongly uphill reaction in a thermodynamic sense. For this reason, the most costly aspect of neural activity is likely to be excitatory synaptic activity in which glutamate opens sodium channels.
Indeed, the action of the sodium-potassium pump is thought to consume a large fraction of the ATP energy budget in the brain (Ames, 2000). In a recent animal experiment, blocking voltagedependent sodium channels substantially reduced the $\mathrm{CBF}$ response (Kida et al., 2001), supporting the idea that the dominant energy consuming process in the brain is recovery from excitatory activity. Finally, there is some evidence that inhibitory activity does not elicit a measurable BOLD response (Waldvogel et al., 2000).

Friston et al. (2000) introduced a simple neurovascular coupling model in which the rate of change of $\mathrm{CBF}$ is proportional to the concentration of a vasoactive agent released by neural activity. In this model, the CBF response is a linear transformation of the neural activity. Using this model, they showed that an observed set of nonlinearities modeled with Volterra kernels could be well described with this more physiological model. This model was also used to explore the effects of nonlinearity in different experimental designs (Mechelli et al., 2001).

One of the goals of modeling the hemodynamic response is to understand the origins of the nonlinearities of the response, and for that purpose, it is useful to have a model that includes a nonlinear transformation from the stimulus pattern $s(t)$ to the CBF response $f(t)$. Such a nonlinearity could arise in the step from $s(t)$ to the neural response $N(t)$, as, for example, in adaptation. In addition, the step from neural activity to $\mathrm{CBF}$ response could be nonlinear, for example, through a ceiling effect on $\mathrm{CBF}$ change. Given our poor understanding of the mechanisms of neurovascular coupling, we take here a simple approach and assume that the nonlinear step is entirely in the transformation from $s(t)$ to $N(t)$, and in the next section we introduce a simple model for this process that includes adaptation. We then assume that both $\mathrm{CBF}$ and $\mathrm{CMRO}_{2}$ are linear convolutions of an impulse response function $h(t)$ with the appropriate measure of neural activity $N(t)$.

A plausible shape for $h(t)$ is a gamma-variate function with a full width at half maximum (FWHM) of about $4 \mathrm{~s}$. For the calculations here we use the form:

$h(t)=\frac{1}{k \tau_{h}(k-1) !}\left(\frac{t}{\tau_{h}}\right)^{k} e^{-t / \tau_{h}}$

with $k=3$. For the calculations in this paper we also add a delay of this response (typically about $1 \mathrm{~s}$ ) to model the observed lag of the hemodynamic response.

The shape $h(t)$ is then scaled to provide the desired amplitude and duration of the impulse response. For this shape, and a desired FWHM of $\tau_{\mathrm{f}}$, the time constant in Eq. (12) is given by the empirical expression $\tau_{\mathrm{h}}=0.242 \tau_{\mathrm{f}}$. The $\mathrm{CBF}$ and $\mathrm{CMRO}_{2}$ responses to activation are then:

$$
\begin{aligned}
& f(t)=1+\left(f_{1}-1\right) h\left(t-\delta t_{f}\right) * N(t) \\
& m(t)=1+\left(m_{1}-1\right) g\left(t-\delta t_{m}\right) * N(t)
\end{aligned}
$$

The symbol * denotes convolution. The parameter $f_{1}$ scales the response shape to the appropriate amplitude and represents the normalized flow increase on the plateau of the $\mathrm{CBF}$ response to a sustained neural activity with unit amplitude. For example, if $N(t)$ is a 30-s block with amplitude 1 , and the model parameters are $f_{1}=1.5$ and $\tau_{\mathrm{f}}=4 \mathrm{~s}$, the CBF response is a smoothed version of the block due to the 4-s-wide smoothing kernel, and on the plateau CBF is increased by $50 \%$. The parameter $\delta t_{f}$ is the delay after the start of the stimulus before the $\mathrm{CBF}$ response begins.

We model the $\mathrm{CMRO}_{2}$ response in Eq. (13) as an independent convolution with potentially independent amplitude, width, and 
delay defined by $\mathrm{g}(\mathrm{t})$. In the calculations here, we assume a coupled response such that the amplitude of the $\mathrm{CMRO}_{2}$ impulse response is given by $\left(m_{1}-1\right)=\left(f_{1}-1\right) / n$, and the width is the same. In this way the steady-state response is constrained to follow the empirical physiological relationship in Eq. (3). However, by introducing a delay $\delta t=\delta t_{f}-\delta t_{m}$ of the CBF response relative to the $\mathrm{CMRO}_{2}$ response, we can introduce interesting dynamics such as an initial dip of the BOLD response. This approach is analogous to the balloon model, where the model is constrained to follow the physiological relationship in Eq. (1) at steady state, but allows substantial range for transient responses.

Fig. 4 illustrates the type of transient features that can result from combining the balloon model with the independent convolution model. The figure shows different dynamic responses of $\mathrm{CBF}, \mathrm{CMRO}_{2}$, and $\mathrm{CBV}$ to the same 20-s uniform block of neural activity. In these calculations, the responses $f(t)$ and $m(t)$ calculated from the independent convolutions were used as input to the balloon model to calculate $v(t)$ and $q(t)$. The first panel shows the response when the viscoelastic time constants of the balloon model are zero, and there is no delay between $f(t)$ and $m(t)$. In the second panel, $\tau_{-}$was increased to $20 \mathrm{~s}$, and in the third panel the impulse response for CBF was delayed by $\delta t=1 \mathrm{~s}$ relative to the $\mathrm{CMRO}_{2}$ response. The BOLD response for the last combination shows both an initial dip and a poststimulus undershoot. The physiological dynamics is also shown as a trajectory in the $q / v$ plane on the right side of Fig. 4, and the BOLD signal is a one-dimensional projection of this two dimensional trajectory.

\section{Modeling the neural response}

As discussed in the previous section, the approach we have adopted is to model the $\mathrm{CBF}$ and $\mathrm{CMRO}_{2}$ responses as linear convolutions with the neural activity $N(t)$, and uses a model for the step from the stimulus $s(t)$ to $N(t)$ that includes the possibility for adaptation. We chose a simple inhibitory feedback system, in which the neural response $N(t)$ is treated as the difference between an excitatory input $s(t)$ and an inhibitory input $I(t)$. The inhibitory input $I(t)$ is driven by the neural response $N(t)$ with a gain factor $\kappa$ and a time constant $\tau_{\mathrm{I}}$. The set of equations is then:

$$
\begin{aligned}
& N(t)=s(t)-I(t) \\
& \frac{\mathrm{d} I}{\mathrm{~d} t}=\frac{\kappa N(t)-I(t)}{\tau_{1}}
\end{aligned}
$$

From these equations, the neural response to a sustained stimulus is an initial peak followed by decay to a lower plateau level, with the difference between the peak and plateau values determined by $\kappa$. As written, these equations are linear, and the initial peak of the response would be balanced by a dip after the end of the stimulus, and such a post-undershoot of the neural response has been observed (Logothetis, 2003). We introduce a nonlinear component, as well as the possibility of a post-stimulus neural undershoot, by introducing a baseline neural activity $N_{0}$ and the requirement that the neural response is a positive quantity (i.e., if the calculated quantity $N_{0}+$ $N(t)<0$, it is replaced by zero). Then if the resting stimulus level is $N_{0}=0$, there is no dip following the end of the stimulus. This is the adaptation pattern originally proposed by Boynton et al. to describe the observed nonlinearities of the BOLD response in the visual cortex (Boynton et al., 1996). On the other hand, if $N_{0}>0$, there will be a post-stimulus undershoot of the neural response. In addition to
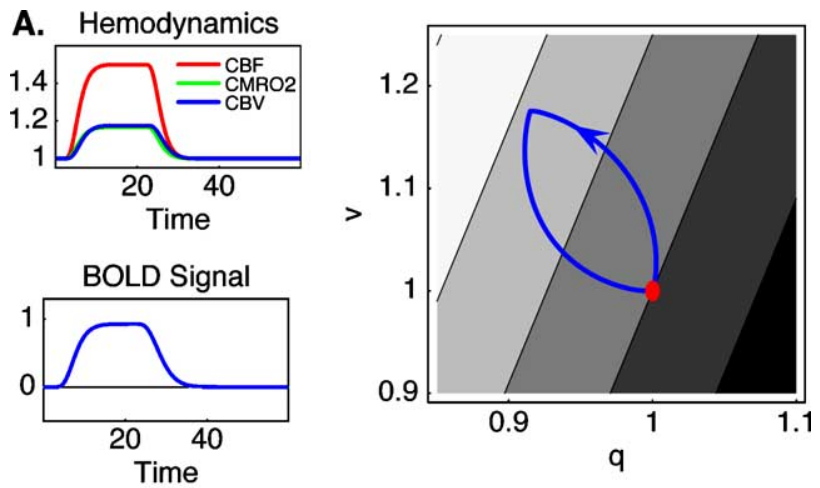

B. Hemodynamics
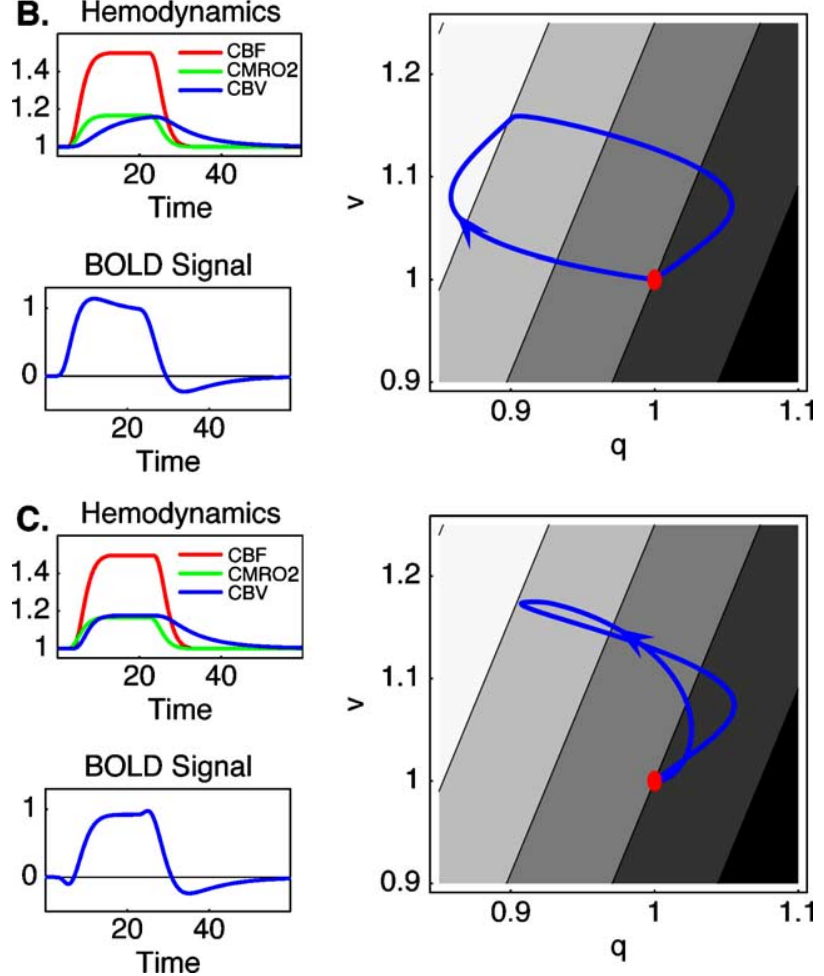

Fig. 4. Transients of the BOLD response due to variations in the timing of the responses of the physiological variables $\mathrm{CBF}, \mathrm{CBV}$, and $\mathrm{CMRO}_{2}$. These variables and the resulting BOLD response are shown on the left. The BOLD response is diagrammed on the right as a trajectory in the $q / v$ plane, where $q$ is the total deoxyhemoglobin and $v$ is the blood volume, both normalized to their baseline values (indicated by a red dot). The BOLD signal intensity is shown as a shaded contour plot in the $q / v$ plane based on Eq. (9). The three sets of figures illustrate: (A) a simple BOLD response in which the physiological changes have similar time courses; (B) a BOLD response with an initial overshoot and poststimulus undershoot due to a slow CBV response $\left(\tau_{+}=\tau_{-}=20 \mathrm{~s}\right)$; and (C) a BOLD response with an initial dip as well, created by adding a 1-s delay of the CBF impulse response relative to the $\mathrm{CMRO}_{2}$ impulse response.

diminishing the response to a sustained stimulus, this model also introduces a "refractory" effect. If two events are presented close together (within $\tau_{\text {I }}$ of each other), then the net response to both events will have less than twice the area of the response to a single event.

This model provides a simple form for introducing a nonlinearity that can be applied to any stimulus pattern: the amplitude of this nonlinear effect is governed by $\kappa$ and the duration of the "refractory" period is determined by $\tau_{\mathrm{I}}$. Fig. 5 shows an example that includes both a two-pulse inhibition experiment and a sustained stimulus. 


\section{Discussion}

\section{Modeling the hemodynamic response}

We have constructed a proposed mathematical framework to link an applied stimulus pattern with the resulting BOLD response. The stimulus pattern drives the neural response, which could exhibit adaptation effects controlled by the parameters $\kappa$ and $\tau_{\mathrm{I}}$. The neural response independently drives the $\mathrm{CBF}$ and $\mathrm{CMRO}_{2}$

A.

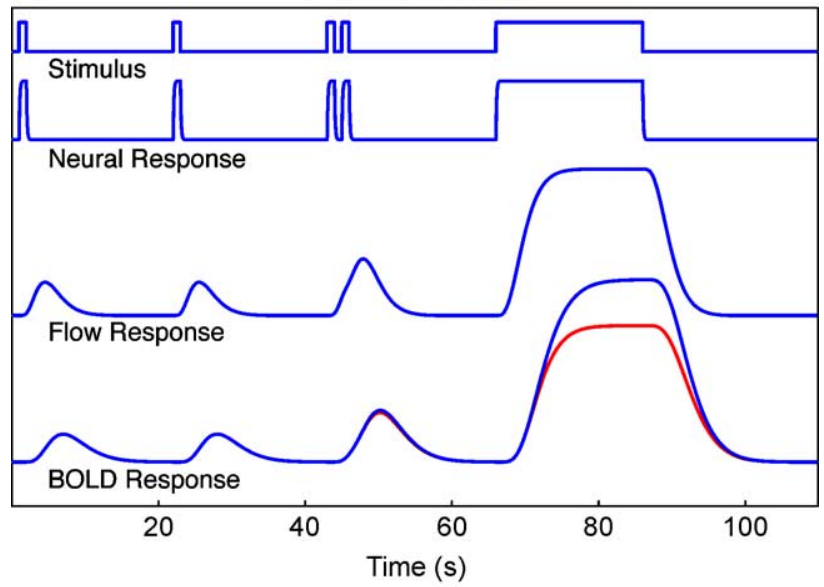

B.

BOLD and Neural Nonlinearity

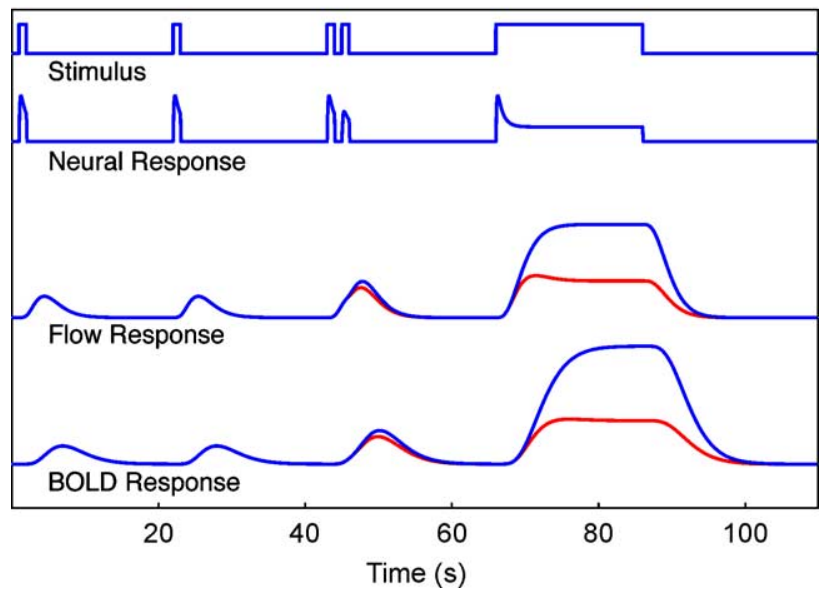

Fig. 5. Nonlinearity of the BOLD response with respect to additivity of overlapped responses. A single event with a 1-s duration (the first stimulus shown) was used to predict the response to a second identical event presented $20 \mathrm{~s}$ later, a pair of identical events separated by a 1-s interstimulus interval, and a block of 20 identical events concatenated to create a continuous stimulation. For the flow and BOLD responses, the predicted curve is shown in blue and the actual calculated curve is shown in red. The calculations show two sources of nonlinearity: (A) with a purely linear neural $(\kappa=0)$ and flow response, the BOLD response still shows a pronounced nonlinearity in the prediction of a sustained response due to the BOLD ceiling effect, with the area of the actual response $22 \%$ lower than the linear prediction. In addition, the area under the response to two pulses close together is slightly reduced by $4 \%$ from twice the area under the response to a single pulse. (B) With a neural response nonlinearity as well ( $\kappa=3$ and $\tau_{\mathrm{I}}=3 \mathrm{~s}$ ), both the flow and BOLD responses exhibit overprediction of the response to a sustained stimulus and a more pronounced "refractory period" in the two pulse experiment (area of the response reduced by $17 \%$ ). responses, treated as simple linear convolutions. The amplitudes of these two responses are defined in terms of a flow amplitude $f_{1}$ and a steady-state $\mathrm{CBF} / \mathrm{CMRO}_{2}$ coupling parameter $n$. A slight delay of the CBF response relative to the $\mathrm{CMRO}_{2}$ response creates an initial dip in the BOLD response. The $\mathrm{CBF}$ and $\mathrm{CMRO}_{2}$ response drives the balloon model, which determines the time course of $\mathrm{CBV}$ and total deoxyhemoglobin. Key parameters for the balloon model are the resting $\mathrm{O}_{2}$ extraction fraction $E_{0}$ and two viscoelastic time constants $\tau_{+}$and $\tau_{-}$. When these time constants are nonzero, the BOLD response exhibits an initial overshoot and a poststimulus undershoot that are not present in the CBF response. Finally, the deoxyhemoglobin and $\mathrm{CBV}$ responses determine the dynamic BOLD response, with the amplitude scaled by the resting venous blood volume fraction $V_{0}$.

A critical feature for modeling the BOLD effect is the relationship between $\mathrm{CBF}$ and $\mathrm{CMRO}_{2}$ changes during activation. Here we have taken the empirical approach, motivated by the experimental data, and modeled this in terms of a single parameter $n$, the ratio of the fractional change in $\mathrm{CBF}$ to the fractional change in $\mathrm{CMRO}_{2}$. A deeper question is to ask why $n$ is not equal to 1 , because this is the central phenomenon underlying the BOLD effect. This is an area of active research (Aubert and Costalat, 2002; Buxton, 2004; Buxton and Frank, 1997; Gjedde et al., 1991, 2002; Hayashi et al., 2003; Hyder et al., 1998; Woo and Hathout, 2001; Zheng et al., 2002), and as this work is refined it can be included as a central step in modeling the path from stimulus to BOLD response.

\section{Transients of the BOLD response}

Notable features of experimental measurements of the BOLD response are the transients, an occasional brief initial dip at the beginning, or the more common prolonged poststimulus undershoot. The poststimulus undershoot often appears as an apparent lowering of the baseline after the first stimulus block, when the undershoot has not fully resolved before the next stimulus block begins. In general, to clearly distinguish the undershoot, the rest block should be longer than the stimulus block. In the framework proposed here, such transients have two distinct sources. The initial dip is modeled as a slight delay (about $1 \mathrm{~s}$ ) of the CBF response compared to the $\mathrm{CMRO}_{2}$ response. The poststimulus undershoot arises in the model because $\mathrm{CBV}$ is slower to recover than $\mathrm{CBF}$ and $\mathrm{CMRO}_{2}$. Then if the oxygen extraction fraction returns to baseline at the end of the stimulus, but the venous blood volume remains elevated, total deoxyhemoglobin will be higher than baseline, reducing the BOLD signal.

The fast response corresponding to the initial dip of the BOLD signal was first detected in optical studies measuring deoxyhemoglobin and oxyhemoglobin changes, and was interpreted as a rapid increase of $E$. However, this interpretation is not fully consistent with these early studies, which found that the fast response period corresponded to an increase of deoxyhemoglobin but no change in oxyhemoglobin (Jones et al., 2001; Malonek and Grinvald, 1996), suggesting a combined change in $\mathrm{CBV}$ and $E$ (Buxton, 2001). In the original version of the balloon model (Buxton et al., 1998c), it was noted that an initial dip of the BOLD signal also could result from a rapid rise in blood volume with no change in $E$. A more recent study, however, found a corresponding initial decrease in oxyhemoglobin in conjunction with an increase in deoxyhemoglobin, more clearly suggesting a change in $E$ (Devor et al., 2003). Although more data are clearly needed to resolve the experimental 
inconsistencies, we have chosen to model the initial dip as a change in $E$, based on the recent experimental work.

\section{Nonlinearity of the $B O L D$ response}

Friston et al. (1998) suggested that a large part of the nonlinearity of the BOLD response arises from the transformation of a $\mathrm{CBF}$ response to the BOLD signal change. In a subsequent study, they showed that the Volterra kernel characterization of experimentally observed nonlinearities could be accounted for with the balloon model plus a linear transformation up to the CBF response, again supporting the idea that the primary nonlinearity is in the transformation from the $\mathrm{CBF}$ to the BOLD response (Friston et al., 2000). In the current model, we also explicitly included an additional nonlinearity of the neural response itself, allowing for an initial sharp response followed by adaptation (a picture originally proposed by Boynton et al., 1996). The two sources of nonlinearity can be distinguished experimentally by whether the nonlinearity is present in just the BOLD response or in both the BOLD and CBF responses. For example, Miller et al. (2001) found that both visual and motor cortices exhibited a nonlinear BOLD response, but only the visual cortex showed a nonlinear CBF response.

The first source is modeled as a nonlinearity of the neural response itself, and this nonlinearity should be present in both the $\mathrm{CBF}$ and BOLD responses. This neural nonlinearity was modeled loosely as an adaptation effect, in which the neural output decreases over time to a lower plateau despite a constant excitatory signal. In the model, we treat this neural output activity as the relevant aspect of neural activity that drives the $\mathrm{CBF}$ and $\mathrm{CMRO}_{2}$ response. As noted, however, current evidence suggests that synaptic activity, rather than spiking activity, is the primary drive of the CBF response, and from energetic considerations excitatory activity is more costly. For this reason, one should be careful of making too literal an interpretation of the neural activity model. The output of the model could represent local spiking activity, if spiking activity is the primary drive for the CBF response. Or, if synaptic activity is the primary drive, the model could represent the input excitatory activity arriving from other neurons that adapt, possibly elsewhere in the brain. Finally, the source of the nonlinearity could be in the CBF response itself, and it is only for convenience that we have isolated the nonlinearity in the neural response and treated the $\mathrm{CBF}$ and $\mathrm{CMRO}_{2}$ responses as linear. To untangle this ambiguity will require combined electrophysiology and ASL measurements. Nevertheless, the model provides a simple mechanism for introducing a nonlinearity in the CBF response.

The second source of nonlinearity is the BOLD ceiling effect. Even an infinite $\mathrm{CBF}$ change could still produce only a finite BOLD response, corresponding to removing all deoxyhemoglobin from the voxel (the curvature of the BOLD signal vs. CBF lines in Fig. 2). This effect produces an overprediction of the amplitude of a long duration stimulus from a short duration stimulus when the flow change due to the short stimulus is substantially weaker than the flow change due to the longer stimulus. Effectively, the extrapolation then covers a large change in $\mathrm{CBF}$, so the curvature shown in Fig. 2 becomes important. On the other hand, if the shorter duration stimulus is long enough for the CBF response to come close to the plateau value of the long duration stimulus, the curvature in Fig. 2 becomes unimportant due to the small range of $\mathrm{CBF}$, and the response should be nearly linear. That is, by this model, the BOLD ceiling effect should introduce a nonlinear response when the shorter stimulus is narrower than the width of the $\mathrm{CBF}$ response, which is about $4 \mathrm{~s}$, in good agreement with experimental data.

These two sources of nonlinearity interact in an interesting way (Miller et al., 2001). If the neural nonlinearity is strong, then the flow response to even a short duration stimulus is strong. In this case the nonlinearity due to the BOLD ceiling effect should be small, because extrapolation from the short stimulus response to the long stimulus response is over a small range of flow change. On the other hand, if the neural nonlinearity is weak, the flow response to the short stimulus can be much weaker than the full flow response to a sustained stimulus. However, in this case the nonlinearity due to the BOLD ceiling effect will be larger. In other words, when one source of nonlinearity is weak, the other tends to be strong. Both produce similar nonlinearities in the BOLD response, but only one of these (the neural nonlinearity) affects the flow response (as illustrated in Fig. 5).

In this paper, we have not discussed nonlinearities of the timing of the response, such as latency or response width, and such effects have been reported. A recent proposal by Behzadi et al. (2004) is a promising approach for accounting for these effects. Instead of treating $\mathrm{CBF}$ as a linear convolution with the neural activity, it is assumed that neural activity releases a vasoactive agent that alters CBF, as in the model of Friston et al. (2000). The difference is that the agent is modeled as acting on the compliance of the vessel. The compliance in turn is treated as a combination of the smooth muscle tension, which can be controlled by the agent, and a fixed elastic component that becomes a more dominant factor in determining compliance when the vessel is expanded. In this way, the same concentration of the agent will have a greater effect on $\mathrm{CBF}$ when the flow is lower, and potentially a different effect on the nonlinearity of the response.

\section{Effect of the physiological baseline on the BOLD response}

Finally, we can use the BOLD signal model to consider the effects of the physiological baseline on the BOLD response. Experiments have found that when baseline CBF is increased by breathing $\mathrm{CO}_{2}$ or administering acetazolamide, the BOLD response is reduced. For example, Brown et al. (2003) found that acetazolamide raised baseline $\mathrm{CBF}$ by $20 \%$ and the BOLD response in the motor cortex with finger tapping was reduced by $35 \%$, but the $\mathrm{CBF}$ change $(\triangle \mathrm{CBF})$ with activation stayed the same.

With $\mathrm{CO}_{2}$ inhalation $\mathrm{CBF}$ increases, but it is thought that $\mathrm{CMRO}_{2}$ remains the same. This means that at this new baseline, the oxygen extraction fraction $E$ must be smaller than it was at the previous baseline, and this implies that there is less deoxyhemoglobin present at the new baseline. The scaling factor $A$ in Eq. (8) is thus reduced, so even if the fractional changes of the physiological quantities were the same, the BOLD signal would be reduced. However, in addition, the experimental data suggest that $\triangle \mathrm{CBF}$ remains constant despite the raised baseline $\mathrm{CBF}$, so the fractional $\mathrm{CBF}$ change is smaller at the elevated baseline, and this will reduce the BOLD response further.

As an example, consider Eq. (8) with $A=0.1$ and an activation that produces a $30 \%$ change in $\mathrm{CBF}(f=1.3)$ and a $10 \%$ change in $\mathrm{CMRO}_{2}(m=1.1)$ from the initial baseline state. If the subject now breathes $\mathrm{CO}_{2}$ that produces a $20 \%$ increase of baseline $\mathrm{CBF}$ with no change in $\mathrm{CMRO}_{2}$, and then the activation experiment produces the same $\mathrm{CBF}$ and $\mathrm{CMRO}_{2}$ changes, the BOLD signal change is predicted to be reduced by $42 \%$, in reasonable agreement with the experimental data. 
The implication of this sensitivity of the BOLD signal to the baseline state is that, potentially, many factors could alter the baseline state of a patient group (e.g., anxiety or vasoactive medications) that could make their BOLD responses significantly different from a healthy population even if the neural responses in the two groups are identical. For this reason, the $\triangle \mathrm{CBF}$ measured with ASL techniques may prove to be a much more robust approach for quantitative fMRI studies.

While these considerations show how changes in the baseline CBF can affect the BOLD response, we have not dealt with the issue of what determines the baseline $\mathrm{CBF}$. A recent theory that requires further development is that $\mathrm{CBF}$ is regulated to maintain a constant ratio of $\mathrm{O}_{2}$ to $\mathrm{CO}_{2}$ at the mitochondria to preserve the thermodynamic free energy available from oxidative metabolism of glucose (Buxton, 2004). The $\mathrm{O}_{2}$ concentration at the mitochondria $\left(\left[\mathrm{O}_{2}\right]\right)$ is determined by $E$, so to increase the diffusive flux of $\mathrm{O}_{2}$ from capillaries to mitochondria, while maintaining $\left[\mathrm{O}_{2}\right]$, requires $E$ to decrease with activation. In addition, increasing $\mathrm{CO}_{2}$ in the blood degrades the $\left[\mathrm{O}_{2}\right] /\left[\mathrm{CO}_{2}\right]$ ratio, and this is again restored by decreasing $E$. Thus, the model predicts that $\mathrm{CBF}$ should increase with $\mathrm{CO}_{2}$, and that an additional increase is required to meet the needs of increased $\mathrm{CMRO}_{2}$. By this model, the additivity of $\mathrm{CBF}$ changes due to activation and $\mathrm{CO}_{2}$ is a special property of $\mathrm{CO}_{2}-$ induced changes in the baseline. Future experiments are needed to test whether this phenomenon also occurs when the baseline CBF is altered by other means.

In conclusion, the proposed mathematical framework captures several features that have been reported in experimental measurements of the BOLD effect. However, this does not mean that all of the physical ideas motivating the model are correct. More detailed experiments using techniques that measure $\mathrm{CBF}, \mathrm{CBV}$, and even field potentials will be necessary to test these basic mechanisms. Nevertheless, the model provides a framework for simulating the BOLD response or for fitting experimental data to quantitatively characterize observed responses.

\section{Acknowledgments}

We would like to thank Joe Mandeville for helpful comments regarding the manuscript. The authors are supported by NIH grants NS-36722 and NS-042069, and by a grant from the Whitaker Foundation.

\section{References}

Ames III, A., 2000. CNS energy metabolism as related to function. Brain Res. Brain Res. Rev. 34, 42-68.

Ances, B.M., Greenberg, J.H., Detre, J.A., 2001. The effects of graded hypercapnia on the activation flow coupling response due to forepaw stimulation in alpha-chloralose anesthetized rats. Brain Res. 911, 82-88.

Attwell, D., Laughlin, S.B., 2001. An energy budget for signaling in the grey matter of the brain. J. Cereb. Blood Flow Metab. 21, 1133-1145.

Aubert, A., Costalat, R., 2002. A model of the coupling between brain electrical activity, metabolism, and hemodynamics: application to the interpretation of functional neuroimaging. NeuroImage 17, 1162-1181.

Bandettini, P.A., Wong, E.C., Hinks, R.S., Tikofsky, R.S., Hyde, J.S., 1992. Time course EPI of human brain function during task activation. Magn. Reson. Med. 25, 390-397.

Behzadi, Y., Restom, K., Liu, T.T., 2004. Modeling the effect of baseline arteriolar compliance on BOLD dynamics. ISMRM 12th Scientific Meeting, Kyoto, Japan.
Birn, R.M., Saad, Z.S., Bandettini, P.A., 2001. Spatial heterogeneity of the nonlinear dynamics in the FMRI BOLD response. NeuroImage 14, $817-826$

Boxerman, J.L., Bandettini, P.A., Kwong, K.K., Baker, J.R., Davis, T.L., Rosen, B.R., Weisskoff, R.M., 1995. The intravascular contribution to fMRI signal change: Monte Carlo modeling and diffusion-weighted studies in vivo. Magn. Reson. Med. 34, 4-10.

Boxerman, J.L., Hamberg, L.M., Rosen, B.R., Weisskoff, R.M., 1995. MR contrast due to intravascular magnetic susceptibility perturbations. Magn. Reson. Med. 34, 555-566.

Boynton, G.M., Engel, S.A., Glover, G.H., Heeger, D.J., 1996. Linear systems analysis of functional magnetic resonance imaging in human V1. J. Neurosci. 16, 4207-4221.

Brown, G.G., Eyler Zorrilla, L.T., Georgy, B., Kindermann, S.S., Wong, E.C., Buxton, R.B., 2003. BOLD and perfusion response to finger-thumb apposition after acetazolamide administration: differential relationship to global perfusion. J. Cereb. Blood Flow Metab. 23, 829-837.

Buckner, R.L., 1998. Event-related fMRI and the hemodynamic response. Hum. Brain Mapp. 6, 373-377.

Buxton, R.B., 2001. The elusive initial dip. NeuroImage 13, 953-958.

Buxton, R.B., 2002. Coupling between CBF and CMRO2 during neuronal activity. In: Tomita, M., Kanno, I., Hamel, E. (Eds.), Brain Activation and CBF Control. Elsevier Science, The Netherlands, pp. 23-32.

Buxton, R.B., 2002. Introduction to Functional Magnetic Resonance Imaging: Principles and Techniques. Cambridge University Press, Cambridge.

Buxton, R.B., 2004. A hypothesis for cerebral blood flow regulation and the origin of the BOLD effect. 12th ISMRM Scientific Meeting, Kyoto, Japan.

Buxton, R.B., Frank, L.R., 1997. A model for the coupling between cerebral blood flow and oxygen metabolism during neural stimulation. J. Cereb. Blood Flow Metab. 17, 64-72.

Buxton, R.B., Luh, W.-M., Wong, E.C., Frank, L.R., Bandettini, P.A., 1998. Diffusion weighting attenuates the BOLD peak signal change but not the post-stimulus undershoot. Sixth Meeting, International Society for Magnetic Resonance in Medicine, Sydney, Australia, pp. 7.

Buxton, R.B., Miller, K., Frank, L.R., Wong, E.C., 1998. BOLD signal dynamics: the balloon model with viscoelastic effects. Sixth Meeting, International Society for Magnetic Resonance in Medicine, Sydney, Australia, pp. 1401.

Buxton, R.B., Wong, E.C., Frank, L.R., 1998. Dynamics of blood flow and oxygenation changes during brain activation: the balloon model. Magn. Reson. Med. 39, 855-864.

Corfield, D.R., Murphy, K., Josephs, O., Adams, L., Turner, R., 2001. Does hypercapnia-induced cerebral vasodilation modulate the hemodynamic response to neural activation? NeuroImage 13, 1207-1211.

Davis, T.L., Kwong, K.K., Weisskoff, R.M., Rosen, B.R., 1998. Calibrated functional MRI: mapping the dynamics of oxidative metabolism. Proc. Natl. Acad. Sci. U. S. A. 95, 1834-1839.

Devor, A., Dunn, A.K., Andermann, M.L., Ulbert, I., Boas, D.A., Dale, A.M., 2003. Coupling of total hemoglobin concentration, oxygenation, and neural activity in rat somatosensory cortex. Neuron $39,353-359$.

Ernst, T., Hennig, J., 1994. Observation of a fast response in functional MR. Magn. Reson. Med., 146-149.

Feng, C.M., Liu, H.L., Fox, P.T., Gao, J.H., 2001. Comparison of the experimental BOLD signal change in event-related fMRI with the balloon model. NMR Biomed. 14, 397-401.

Fox, P.T., Raichle, M.E., 1986. Focal physiological uncoupling of cerebral blood flow and oxidative metabolism during somatosensory stimulation in human subjects. Proc. Natl. Acad. Sci. U. S. A. 83, 1140-1144.

Frahm, J., Krüger, G., Merboldt, K.-D., Kleinschmidt, A., 1996. Dynamic uncoupling and recoupling of perfusion and oxidative metabolism during focal activation in man. Magn. Reson. Med. 35, 143-148.

Friston, K.J., 2002. Bayesian estimation of dynamical systems: an application to fMRI. NeuroImage 16, 513-530.

Friston, K.J., Josephs, O., Rees, G., Turner, R., 1998. Non-linear event related responses in fMRI. Magn. Reson. Med. 39, 41-52. 
Friston, K.J., Mechelli, A., Turner, R., Price, C.J., 2000. Nonlinear responses in fMRI: the Balloon model, Volterra kernels, and other hemodynamics. NeuroImage 12, 466-477.

Gjedde, A., Ohta, S., Kuwabara, H., Meyer, E., 1991. Is oxygen diffusion limiting for bloodbrain transfer of oxygen? In: Lassen, N., Ingvar, D., Raichle, M., et al. (Eds.), Brain Work and Mental Activity. Alfred Benzon Symposium, Copenhagen, pp. 177-184.

Gjedde, A., Poulsen, P.H., Ostergaard, L., 1999. On the oxygenation of hemoglobin in the human brain. Adv. Exp. Med. Biol. 471, 67-81.

Gjedde, A., Marrett, S., Vafaee, M., 2002. Oxidative and nonoxidative metabolism of excited neurons and astrocytes. J. Cereb. Blood Flow Metab. 22, 1-14.

Glover, G.H., 1999. Deconvolution of impulse response in event-related fMRI. NeuroImage 9, 416-429.

Grubb, R.L., Raichle, M.E., Eichling, J.O., Ter-Pogossian, M.M., 1974. The effects of changes in $\mathrm{PaCO}_{2}$ on cerebral blood volume, blood flow, and vascular mean transit time. Stroke 5, 630-639.

Hayashi, T., Watabe, H., Kudomi, N., Kim, K.M., Enmi, J., Hayashida, K., Iida, H., 2003. A theoretical model of oxygen delivery and metabolism for physiologic interpretation of quantitative cerebral blood flow and metabolic rate of oxygen. J. Cereb. Blood Flow Metab. 23, 1314-1323.

Hess, A., Stiller, D., Kaulisch, T., Heil, P., Scheich, H., 2000. New insights into the hemodynamic blood oxygenation level-dependent response through combination of functional magnetic resonance imaging and optical recording in gerbil barrel cortex. J. Neurosci. 20, 3328-3338.

Hoge, R.D., Atkinson, J., Gill, B., Crelier, G.R., Marrett, S., Pike, G.B., 1999. Linear coupling between cerebral blood flow and oxygen consumption in activated human cortex. Proc. Natl. Acad. Sci. U. S. A. 96, 9403-9408.

Hu, X., Le, T.H., Ugurbil, K., 1997. Evaluation of the early response in fMRI in individual subjects using short stimulus duration. Magn. Reson. Med. 37, 877-884.

Huettel, S.A., McCarthy, G., 2001. Regional differences in the refractory period of the hemodynamic response: an event-related fMRI study. NeuroImage 14, 967-976.

Hyder, F., Shulman, R.G., Rothman, D.L., 1998. A model for the regulation of cerebral oxygen delivery. J. Appl. Physiol. 85, 554-564.

Jones, M., Berwick, J., Johnston, D., Mayhew, J., 2001. Concurrent optical imaging spectroscopy and laser-Doppler flowmetry: the relationship between blood flow, oxygenation, and volume in rodent barrel cortex. NeuroImage 13, 1002-1015.

Kastrup, A., Li, T.Q., Kruger, G., Glover, G.H., Moseley, M.E., 1999. Relationship between cerebral blood flow changes during visual stimulation and baseline flow levels investigated with functional MRI. NeuroReport 10, 1751-1756.

Kastrup, A., Kruger, G., Neumann-Haefelin, T., Glover, G.H., Moseley, M.E., 2002. Changes of cerebral blood flow, oxygenation, and oxidative metabolism during graded motor activation. NeuroImage 15, 74-82.

Kida, I., Hyder, F., Behar, K.L., 2001. Inhibition of voltage-dependent sodium channels suppresses the functional magnetic resonance imaging response to forepaw somatosensory activation in the rodent. J. Cereb. Blood Flow Metab. 21, 585-591.

Kim, S.G., Rostrup, E., Larsson, H.B.W., Ogawa, S., Paulson, O.B., 1999. Determination of relative $\mathrm{CMRO} 2$ from $\mathrm{CBF}$ and BOLD changes: significant increase of oxygen consumption rate during visual stimulation. Magn. Reson. Med. 41, 1152-1161.

Kruger, G., Kleinschmidt, A., Frahm, J., 1996. Dynamic MRI sensitized to cerebral blood oxygenation and flow during sustained activation of human visual cortex. Magn. Reson. Med. 35, 797-800.

Kuwabara, H., Ohta, S., Brust, P., Meyer, E., Gjedde, A., 1992. Density of perfused capillaries in living human brain during functional activation. Prog. Brain Res. 91, 209-215.

Kwong, K.K., Belliveau, J.W., Chesler, D.A., Goldberg, I.E., Weisskoff, R.M., Poncelet, B.P., Kennedy, D.N., Hoppel, B.E., Cohen, M.S., Turner, R., et al., 1992. Dynamic magnetic resonance imaging of human brain activity during primary sensory stimulation. Proc. Natl. Acad. Sci. U. S. A. $89,5675-5679$.
Lauritzen, M., 2001. Relationship of spikes, synaptic activity, and local changes of cerebral blood flow. J. Cereb. Blood Flow Metab. 21, $1367-1383$.

Lauritzen, M., Gold, L., 2003. Brain function and neurophysiological correlates of signals used in functional neuroimaging. J. Neurosci. 23, 3972-3980.

Lennie, P., 2003. The cost of cortical computation. Curr. Biol. 13, 493-497.

Li, T.Q., Moseley, M.E., Glover, G., 1999. A FAIR study of motor cortex activation under normo- and hypercapnia induced by breath challenge. NeuroImage 10, 562-569.

Li, T.Q., Kastrup, A., Moseley, M.E., Glover, G.H., 2000. Changes in baseline cerebral blood flow in humans do not influence regional cerebral blood flow response to photic stimulation. J. Magn. Reson. Imaging 12, 757-762.

Lindauer, U., Royl, G., Leithner, C., Kuhl, M., Gold, L., Gethmann, J., Kohl-Bareis, M., Villringer, A., Dirnagl, U., 2001. No evidence for early decrease in blood oxygenation in rat whisker cortex in response to functional activation. NeuroImage 13, 988-1001.

Logothetis, N.K., 2002. The neural basis of the blood-oxygen-leveldependent functional magnetic resonance imaging signal. Philos. Trans. R. Soc. London, Ser. B Biol. Sci. 357, 1003-1037.

Logothetis, N.K., 2003. The underpinnings of the BOLD functional magnetic resonance imaging signal. J. Neurosci. 23, 3963-3971.

Logothetis, N.K., Pauls, J., Augath, M., Trinath, T., Oeltermann, A., 2001. Neurophysiological investigation of the basis of the fMRI signal. Nature 412, 150-157.

Lu, H., Golay, X., Pekar, J.J., Van Zijl, P.C., 2003. Functional magnetic resonance imaging based on changes in vascular space occupancy. Magn. Reson. Med. 50, 263-274.

Malonek, D., Grinvald, A., 1996. Interactions between electrical activity and cortical microcirculation revealed by imaging spectroscopy: implications for functional brain mapping. Science 272, 551-554.

Mandeville, J.B., Marota, J.J.A., Kosofsky, B.E., Keltner, J.R., Weissleder, R., Rosen, B.R., Weisskoff, R.M., 1998. Dynamic functional imaging of relative cerebral blood volume during rat forepaw stimulation. Magn. Reson. Med. 39, 615-624.

Mandeville, J.B., Marota, J.J.A., Ayata, C., Moskowitz, M.A., Weisskoff, R.M., Rosen, B.R., 1999a. MRI measurement of the temporal evolution of relative $\mathrm{CMRO} 2$ during rat forepaw stimulation. Magn. Reson. Med. 42, 944-951.

Mandeville, J.B., Marota, J.J.A., Ayata, C., Zaharchuk, G., Moskowitz, M.A., Rosen, B.R., Weisskoff, R.M., 1999b. Evidence of a cerebrovascular post-arteriole Windkessel with delayed compliance. J. Cereb. Blood Flow Metab. 19, 679-689.

Marrett, S., Gjedde, A., 1997. Changes of blood flow and oxygen consumption in visual cortex of living humans. Adv. Exp. Med. Biol. 413, 205-208.

Mechelli, A., Price, C.J., Friston, K.J., 2001. Nonlinear coupling between evoked rCBF and BOLD signals: a simulation study of hemodynamic responses. NeuroImage 14, 862-872.

Menon, R.S., Ogawa, S., Strupp, J.P., Anderson, P., Ugurbil, K., 1995. BOLD based functional MRI at 4 tesla includes a capillary bed contribution: echo-planar imaging correlates with previous optical imaging using intrinsic signals. Magn. Reson. Med. 33, 453-459.

Mildner, T., Norris, D.G., Schwarzbauer, C., Wiggins, C.J., 2001. A qualitative test of the balloon model for BOLD-based MR signal changes at 3T. Magn. Reson. Med. 46, 891-899.

Miller, K.L., Luh, W.M., Liu, T.T., Martinez, A., Obata, T., Wong, E.C., Frank, L.R., Buxton, R.B., 2001. Nonlinear temporal dynamics of the cerebral blood flow response. Hum. Brain Mapp. 13, 1-12.

Obata, T., Liu, T.T., Miller, K.L., Luh, W.M., Wong, E.C., Frank, L.R., Buxton, R.B., 2004. Discrepancies between BOLD and flow dynamics in primary and supplementary motor areas: application of the balloon model to the interpretation of BOLD transients. NeuroImage 21, 144-153.

Ogawa, S., Lee, T.M., Kay, A.R., Tank, D.W., 1990. Brain magnetic resonance imaging with contrast dependent on blood oxygenation. Proc. Natl. Acad. Sci. U. S. A. 87, 9868-9872. 
Ogawa, S., Menon, R.S., Tank, D.W., Kim, S.-G., Merkle, H., Ellerman, J.M., Ugurbil, K., 1993. Functional brain mapping by blood oxygenation level-dependent contrast magnetic resonance imaging: a comparison of signal characteristics with a biophysical model. Biophys. J. 64, $803-812$.

Robson, M.W., Dorosz, J.L., Gore, J.C., 1998. Measurements of the temporal fMRI response of the human auditory cortex to trains of tones. NeuroImage 7, 185-198.

Seitz, R.J., Roland, P.E., 1992. Vibratory stimulation increases and decreases the regional cerebral blood flow and oxidative metabolism: a positron emission tomography (PET) study. Acta Neurol. Scand. 86, 60-67.

Toronov, V., Walker, S., Gupta, R., Choi, J.H., Gratton, E., Hueber, D., Webb, A., 2003. The roles of changes in deoxyhemoglobin concentration and regional cerebral blood volume in the fMRI BOLD signal. NeuroImage 19, 1521-1531.

Vasquez, A.L., Noll, D.C., 1998. Nonlinear aspects of the BOLD response in functional MRI. NeuroImage 7, 108-118.
Waldvogel, D., van Gelderen, P., Muellbacher, W., Ziemann, U., Immisch, I., Hallett, M., 2000. The relative metabolic demand of inhibition and excitation. Nature 406, 995-998.

Wong, E.C., Buxton, R.B., Frank, L.R., 1997. Implementation of quantitative perfusion imaging techniques for functional brain mapping using pulsed arterial spin labeling. NMR Biomed. 10, 237-249.

Woo, J.H., Hathout, G.M., 2001. Systems analysis of functional magnetic resonance imaging data using a physiologic model of venous oxygenation. J. Cereb. Blood Flow Metab. 21, 517-528.

Yablonsky, D.A., Haacke, E.M., 1994. Theory of NMR signal behavior in magnetically inhomogeneous tissues: the static dephasing regime. Magn. Reson. Med. 32, 749-763.

Yacoub, E., Hu, X., 2001. Detection of the early decrease in fMRI signal in the motor area. Magn. Reson. Med. 45, 184-190.

Zheng, Y., Martindale, J., Johnston, D., Jones, M., Berwick, J., Mayhew, J., 2002. A model of the hemodynamic response and oxygen delivery to brain. NeuroImage 16, 617-637. 